\title{
Are the earlier disease onset and uveitis affected by the HLA-B*27 subtypes in ankylosing spondylitis? Comments on the article by Silmani et al.
}

\author{
Sasan Fallahi ${ }^{1,2}$ iD \\ Received: 28 February 2021 / Revised: 28 February 2021 / Accepted: 5 March 2021 / Published online: 25 March 2021 \\ (C) International League of Associations for Rheumatology (ILAR) 2021
}

To editor:

I read the interesting paper entitled Spondyloarthritis in North Africa: an update which has been published by Silmani et al. in clinical rheumatology [1]. I decided to critique a small part of it briefly, which is about the clinical relevance of HLA$B * 27$ polymorphisms in patients with ankylosing spondylitis (AS) in Asia. They included in their paper: "This is contrary to the findings reported for Asian populations, for whom HLA$B * 27$ polymorphisms may affect disease phenotype, particularly $B * 2704$ and $B * 27015$, which are associated with earlier disease onset and more frequent uveitis than $B * 2705$ among Asian patients" [1]. One of the articles cited by Salmani and her colleagues on this subject is the article by Fallahi et al. [2]. It should be noted that in Fallahi et al.'s study, none of the patients with AS was HLA-B*27015 positive at all and the HLA-B27-positive polymorphisms were as follows: $48.4 \%$ $\mathrm{B} * 2705,42.6 \% \mathrm{~B} * 2702,5.7 \% \mathrm{~B} * 2704$, and $3.3 \% \mathrm{~B} * 2707$. As can be seen, HLA-B*2704 and HLA-B*2707 subtypes account for a small percentage. Also, earlier disease onset and uveitis were not more frequent significantly than the more common subtypes (including HLA-B*2705 and HLA$B * 2702$ ) in this study. However, clinical trends toward less dorsal kyphosis and less decrease in cervical slope (without statistically significance) were observed in patients with $B * 2704$ and $B * 2707$ [2]. In conclusion, uveitis and earlier onset of AS may be more frequent for HLA-B*2704 or HLA-B*27015 among China and Far East population

Sasan Fallahi

sfallahi@sina.tums.ac.ir; drsfallahi@gmail.com

1 Internal Medicine Division, Baharloo Hospital, Tehran University of Medical Sciences, Behdari street, South Kargar Street, Tehran, Iran

2 Rheumatology Research Center, Tehran University of Medical Sciences, Tehran, Iran according to some researchers $[3,4]$. However, this cannot be extended to all parts of Asia, especially Iran or Middle East Asia.

\section{Declarations}

Conflict of interest The author declares no conflict of interest.

\section{References}

1. Slimani S, Hamdi W, Nassar K, Kalla AA (2021) Spondyloarthritis in North Africa: an update. Clin Rheumatol. https://doi.org/10.1007/ s10067-021-05630-w

2. Fallahi S, Mahmoudi M, Nicknam MH et al (2013) Effect of $\mathrm{HLAB} * 27$ and its subtypes on clinical manifestations and severity of ankylosing spondylitis in Iranian patients. Iran J Allergy Asthma Immunol 12:321-330

3. Mou Y, Zhang P, Li Q et al (2015) Clinical features in juvenile onset ankylosing spondylitis patients carrying different B27 subtypes. Biomed Res Int 2015:594878

4. Qi J, Li Q, Lin Z, Liao Z, Wei Q, Cao S, Rong J, Hu Z, Yang M, Zhang Y, Lv Q, Huang J, Pan Y, Wu Y, Jin O, Li T, Gu J (2013) Higher risk of uveitis and dactylitis and older age of onset among ankylosing spondylitis patients with HLA-B*2705 than patients with HLA-B*2704 in the Chinese population. Tissue Antigens 82:380386

Publisher's note Springer Nature remains neutral with regard to jurisdictional claims in published maps and institutional affiliations. 